\title{
Clinical and Molecular Data Define a Diagnosis of Arrhythmogenic Cardiomyopathy in a Carrier of a Brugada-Syndrome-Associated PKP2 Mutation
}

\author{
Simone Persampieri ${ }^{1,+}$, Chiara Assunta Pilato ${ }^{2, \dagger}$, Elena Sommariva ${ }^{2, *}$, \\ Angela Serena Maione ${ }^{2}$, Ilaria Stadiotti ${ }^{2}$, Antonio Ranalletta ${ }^{1}$, Margherita Torchio ${ }^{3}{ }^{(1)}$, \\ Antonio Dello Russo 1,4 (D), Cristina Basso ${ }^{5}$, Giulio Pompilio ${ }^{2,6}$, Claudio Tondo 1,6 \\ and Michela Casella ${ }^{1,4}$ (iD) \\ 1 Cardiac Arrhythmia Research Centre, Centro Cardiologico Monzino IRCCS, 20141 Milan, Italy; \\ simone.persampieri@gmail.com (S.P.); remoantonio_ranalletta@hotmail.it (A.R.); \\ antonio.dellorusso@gmail.com (A.D.R.); claudio.tondo@ccfm.it (C.T.); michela.casella@ccfm.it (M.C.) \\ 2 Vascular Biology and Regenerative Medicine Unit, Centro Cardiologico Monzino IRCCS, 20141 Milan, Italy; \\ chiarapilato91@gmail.com (C.A.P.); angela.maione@ccfm.it (A.S.M.); ilaria.stadiotti@ccfm.it (I.S.); \\ giulio.pompilio@ccfm.it (G.P.) \\ 3 Istituto Auxologico Italiano, IRCCS, Center for Cardiac Arrhythmias of Genetic Origin and Laboratory of \\ Cardiovascular Genetics, 20149 Milan, Italy; m.torchio@auxologico.it \\ 4 Cardiology and Arrhythmology Clinic, University Hospital "Ospedali Riuniti Umberto I-Lancisi - Salesi”, \\ 60020 Ancona, Italy \\ 5 Cardiovascular Pathology Unit, Department of Cardiac-Thoracic-Vascular Sciences and Public Health, \\ University of Padua, 35128 Padua, Italy; cristina.basso@unipd.it \\ 6 Department of Clinical Sciences and Community Health, Università degli Studi di Milano, 20122 Milan, Italy \\ * Correspondence: esommariva@ccfm.it; Tel.: +39-02-5800-2026 \\ + These authors contributed equally to this work.
}

Received: 7 April 2020; Accepted: 18 May 2020; Published: 20 May 2020

\begin{abstract}
Plakophilin-2 (PKP2) is the most frequently mutated desmosomal gene in arrhythmogenic cardiomyopathy (ACM), a disease characterized by structural and electrical alterations predominantly affecting the right ventricular myocardium. Notably, ACM cases without overt structural alterations are frequently reported, mainly in the early phases of the disease. Recently, the PKP2 p.S183N mutation was found in a patient affected by Brugada syndrome (BS), an inherited arrhythmic channelopathy most commonly caused by sodium channel gene mutations. We here describe a case of a patient carrier of the same BS-related PKP2 p.S183N mutation but with a clear diagnosis of ACM. Specifically, we report how clinical and molecular investigations can be integrated for diagnostic purposes, distinguishing between ACM and BS, which are increasingly recognized as syndromes with clinical and genetic overlaps. This observation is fundamentally relevant in redefining the role of genetics in the approach to the arrhythmic patient, progressing beyond the concept of "one mutation, one disease", and raising concerns about the most appropriate approach to patients affected by structural/electrical cardiomyopathy. The merging of genetics, electroanatomical mapping, and tissue and cell characterization summarized in our patient seems to be the most complete diagnostic algorithm, favoring a reliable diagnosis.
\end{abstract}

Keywords: Brugada syndrome; arrhythmogenic cardiomyopathy; PKP2; endomyocardial biopsy; cardiac mesenchymal stromal cells; mutation; diagnosis; functional studies 


\section{Introduction}

Arrhythmogenic cardiomyopathy (ACM) and Brugada syndrome (BS) are inherited arrhythmia diseases that lead to sudden cardiac death. ACM hearts are characterized by ventricular structural alterations showing a progressive fibro-fatty infiltration associated with ventricular mechanical and electrical dysfunction. Mutations in different genes, mainly desmosomal, have been implicated in ACM pathogenesis, and most reside in the gene coding for Plakophilin-2 (PKP2) [1]. BS is a genetic channelopathy characterized by ST-segment elevation of coved morphology in right precordial electrocardiogram (ECG) leads, increased risk of ventricular tachycardia, and fibrillation in the absence of cardiac structural disease [2]. Mutations in the SCN5A gene, coding for the main cardiac sodium channel, account for approximately $20 \%-25 \%$ of genotype-positive subjects, and overall only $25 \%-30 \%$ of BS patients have a known genetic defect [3].

The intercalated disc hosts a common protein interacting network, the connexome, that includes molecules of the desmosome and the voltage-gated sodium channel (VGSC) complex. According to this, if the molecular substrates (desmosomes and VGSC) are part of a common network, BS and ACM should also share some common features. It is estimated that as many as $70 \%$ of the mutations linked to familial ACM are in the gene coding for PKP2, a $98 \mathrm{kDa}$ desmosomal protein that interacts with plakoglobin, desmoplakin, and the desmosomal cadherins via its amino-terminal domain [4]. Mutations in PKP2 may therefore destabilize the desmosome and result in arrhythmias and structural alteration simultaneously.

Although a general phenotypic distinction exists between the two pathologies, imaging and histopathological data support the notion that BS is not purely arrhythmogenic but includes, in some cases, structural anomalies [5]. Molecular data demonstrated that arrhythmias in ACM are consequent not only to tissue alterations but also to changes in the intercalated disc subdomain, including desmosomes, connexins, and sodium channels [6]. Additionally, from a genetic point of view, overlapping between ACM and BS was reported. In the continuing search for new causative gene variants in genetically-negative patients, researchers identified SCN5A mutations in some ACM patients [7], and PKP2 mutations were associated with BS [8]. The two diseases seem to overlap in more than one aspect and a deeper analysis of every patient is required. Herein, we present a patient diagnosed with ACM, in whom a PKP2 mutation, known to be causative for BS, was found. In particular, we report how molecular data (based on the use of cardiac mesenchymal stromal cells (C-MSCs) as an ACM in vitro model [9]) could confirm the clinical correct diagnosis.

\section{Materials and Methods}

\subsection{Ethical Statement}

This study complied with the Declaration of Helsinki and was approved by the Centro Cardiologico Monzino-IRCCS Ethic Committee. Written consent was signed by participating patients.

\subsection{Genotype Analysis}

DNA was extracted from blood. Next-generation sequencing was performed (Illumina NextSeq, San Diego, CA, USA) with the TruSight ${ }^{\mathrm{TM}}$ Cardio Sequencing Kit. The alignment of sequence reads to reference human genome (GRCh37/hg19) was performed using GATK software (the GATK software is available as an open-source framework on The Broad Institute's website). Variants in DSC2, DSG2, DSP , PKP2, JUP, TMEM43, RYR2, PLN, SCN5A, and LMNA were filtered with Wannovar and classified according to [10]. Pathogenic mutations were confirmed by Sanger sequencing.

\subsection{Cardiac Mesenchymal Stromal Cell isolation}

Cells were obtained from patient endomyocardial biopsies, as previously described [11]. 


\subsection{Oil Red O Staining}

C-MSCs were cultured in adipogenic medium (as in [11]) for three days, fixed with $4 \%$ paraformaldehyde (PFA) and neutral lipids were visualized by Oil Red O (ORO) staining. Pictures were captured with an Axiovert microscope (Zeiss, Oberkochen, Germany) and quantified with AxioVision Rel.4.8. (Zeiss, Oberkochen, Germany).

\subsection{Real-Time PCR}

Total RNA was extracted using Trizol (ThermoFisher Scientific, Waltham, MS, USA) and reverse transcribed with SuperscriptIII First-Strand Synthesis SuperMix (Invitrogen, Carlsbad, CA, USA). Quantitative real time-polymerase chain reaction (qRT-PCR) was performed in duplicate using $10 \mathrm{ng}$ of cDNA, the iTaq Universal SYBR Green Supermix (Bio-Rad, Hercules, California, United States) and the following primers:

PLIN1-FW: 5'-CATTGAGAAGGTGGTGGAGTA-3'

PLIN1-REV: 5'-CTTGGCCTTGGGAGACTT-3'

$P P A R \gamma$-FW: $5^{\prime}$-ACATAAAGTCCTTCCCGCTGACCA-3'

PPAR $\gamma$-REV : $5^{\prime}$-AAACTGGCAGCCCTGAAAGATGC $-3^{\prime}$

GAPDH- $F W: 5^{\prime}$ - ATGTTCGTCATGGGTGTGAA-3'

GAPDH- REV: 5' - GTCTTCTGGGTGGCAGTGAT-3'

\subsection{Western Blot Analysis}

C-MSC proteins were separated and transferred as in [9]. The membrane was incubated with primary antibodies against Plakophilin-2 (PKP2; BD Transduction Laboratories; Franklin Lakes, NJ, USA; 1:500), and Glyceraldehyde 3-phosphate dehydrogenase (GAPDH; Santa Cruz Biotechnology; Dallas, TX, USA; 1:2000). After incubation with proper secondary chemiluminescent antibodies, the signal was acquired and quantified using Alliance software (version 1D MAX, UVITEC, Cambridge, England, UK).

\subsection{Immunofluorescence}

After fixation with $4 \%$ paraformaldehyde (PFA), cells were incubated with primary antibodies against plakoglobin (PG; Sigma-Aldrich; St. Louis, Missouri, United States; 1:100) and peroxisome proliferator-activated receptor $\gamma$ (PPAR- $\gamma$; Santa Cruz Biotechnology; Dallas, TX, USA; 1:100). Cells were incubated with the appropriate Alexa 488-conjugated secondary antibody. Cell nuclei were stained with Hoechst 33342. Pictures were acquired with an Apotome microscope (Zeiss, Oberkochen, Germany). The positive cell number was normalized to the nuclei number.

\subsection{Statistical Analysis}

Statistics were performed using GraphPad Prism software (version 5 San Diego, CA, USA). Comparison between groups was performed using two-tailed Student's $t$-tests. Results were considered statistically significant for $p<0.05$.

\section{Results}

\subsection{Clinical Data}

A 41-year-old man, known for a history of premature ventricular complexes (PVCs) since 2009, with no prior history of cardiac diseases and no family history of sudden death, was admitted to our department in 2016. A basal ECG showed sinus bradycardia, nonspecific repolarization abnormalities. Previous echocardiogram and cardiac magnetic resonance (MRI) showed cardiac biventricular dysfunction with enlargement of the right-side chambers. No areas of late gadolinium enhancement or lipomatous infiltration were evident. A two-dimensional echocardiogram at admission showed 
biventricular dilation (left ventricular end-diastolic volume (LVEDV), $80 \mathrm{~mL} / \mathrm{m}^{2}$; right ventricular end-diastolic basal diameter, $45 \mathrm{~mm}$ ) and mild biventricular dysfunction (left ventricular ejection fraction (LVEF), 50\%; tricuspid annular plane systolic excursion (TAPSE), $19 \mathrm{~mm}$; right ventricular fractional area change (RVFAC), 21\%), with no relevant valvular abnormalities. A cardiac MRI was performed again during hospitalization (Figure 1A) and showed biventricular dilation (LVEDV, $125.8 \mathrm{~mL} / \mathrm{m}^{2}$; right ventricular end-diastolic volume (RVEDV), $171 \mathrm{~mL} / \mathrm{m}^{2}$ ), mild biventricular dysfunction (LVEF, 50\%; right ventricular ejection fraction (RVEF), 37\%), right ventricle diffuse hypokinesia with basal right ventricle outflow tract (RVOT) akinesia, and areas suspicious for adipose infiltration at the apex of the right ventricle and in the basal segments of the anterior wall of the left ventricle. A Holter ECG showed 3500 premature ventricular contractions (PVCs), with 223 couplets and 13 triplets. No sustained ventricular arrhythmias were evoked by programmed stimulation (electrophysiological study (EPS)). During the EPS, three different PVC morphologies were recorded: one with RVOT morphology and two with left-side origins. According to the substrate analysis and the RVOT morphology of the PVC, pace mapping was performed, and finally radiofrequency was applied to ablate the origin of the PVC. Electroanatomical mapping (Figure 1B) showed unipolar low-voltage areas in the RVOT, which were suggestive of epicardial scarring [12]. In the same area, an endocardial cardiac biopsy was performed, which was sent for histological analysis. The results confirmed the tissue pattern was compatible with the diagnosis of ACM (fibro-fatty substitution, Figure 1C). The ACM diagnosis was based on one major MRI criterion, one minor arrhythmia criterion, and one minor wall-tissue characterization criterion [13]. An implantable cardioverter-defibrillator (ICD) was therefore implanted. The patient was then discharged with sotalol therapy. The genetic screening by next-generation sequencing of the genes DSC2, DSG2, DSP , PKP2, JUP, TMEM43, RYR2, $P L N$, and LMNA revealed the presence of the PKP2 gene in exon 3 of the c.548G $>$ A mutation that causes p.S183N substitution. This rare variant was previously described as causative of BS [8], but has never been associated with ACM. This result raised the suspicion of a misdiagnosis and the patient was recalled to perform an ajmaline test. No type 1 Brugada ECG pattern was evoked (Figure 1D) and the diagnosis of BS was ruled out. During three years of follow-up, no recurrent arrhythmias were evident from remote monitoring or ambulatory visits.

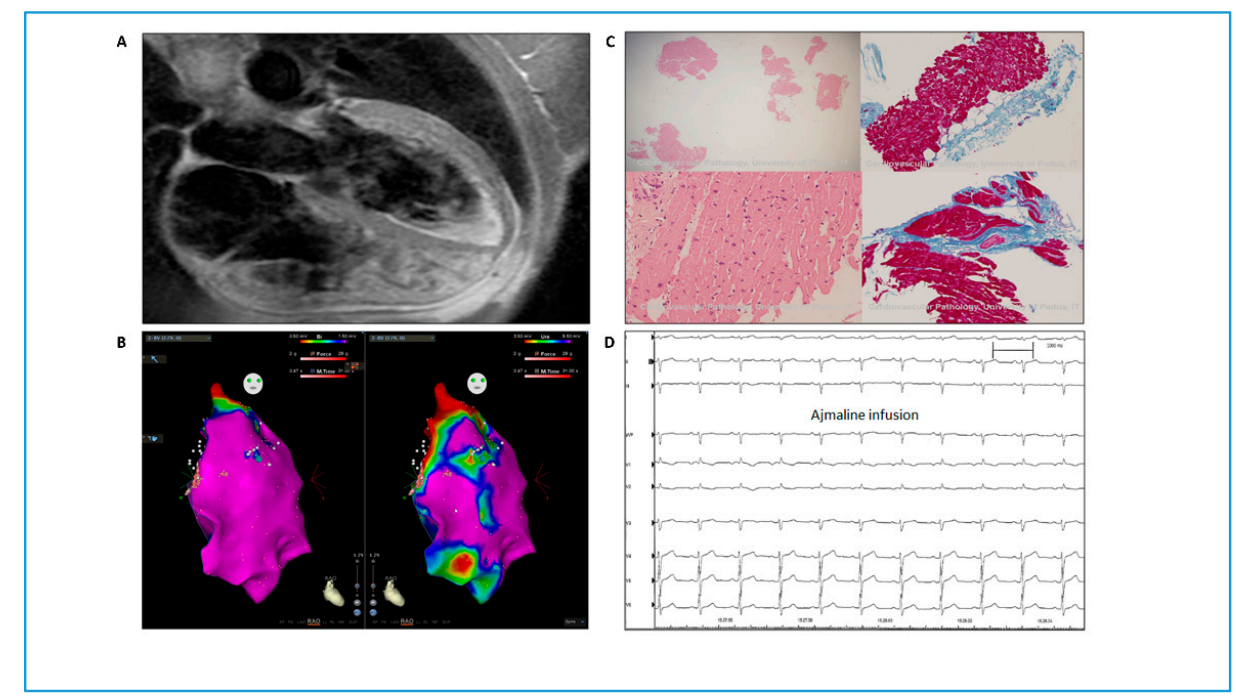

Figure 1. Clinical data are not compatible with BS phenotypes. (A) Cardiac magnetic resonance showing biventricular dilation and areas suspicious for adipose infiltration at the apex of the right ventricle (RV). (B) Bipolar (left) and unipolar (right) electroanatomical mapping of the RV of the patient. Unipolar voltage mapping showed low-voltage areas in the RV outflow tract. (C) Endomyocardial biopsy showing fibro-fatty substitution, compatible with arrhythmogenic cardiomyopathy diagnosis. (D) $1 \mathrm{mg} / \mathrm{kg}$ of ajmaline was administered to the patient over $10 \mathrm{~min}$ followed by $5 \mathrm{~min}$ of wash-out observation: no type-1 BS electrocardiographic pattern was induced. BS, Brugada syndrome. 
A 28-year-old athlete with a clinical and genetic diagnosis of BS (heterozygous c.4867C $>$ T, p.Arg1623X in the SCN5A gene), who previously underwent cardiac biopsy to rule out ACM, was used for comparative analysis.

\subsection{Molecular Data}

C-MSCs were isolated from endomyocardial biopsies obtained from the ACM and BS patients and used to study their molecular phenotypes. As reported in the literature, PKP2 is expressed less in C-MSC isolated from ACM patients than in healthy controls [9]. Accordingly, the level of PKP2 in the ACM patient's C-MSCs was lower than that of the C-MSCs obtained from the BS patient (Figure 2A).

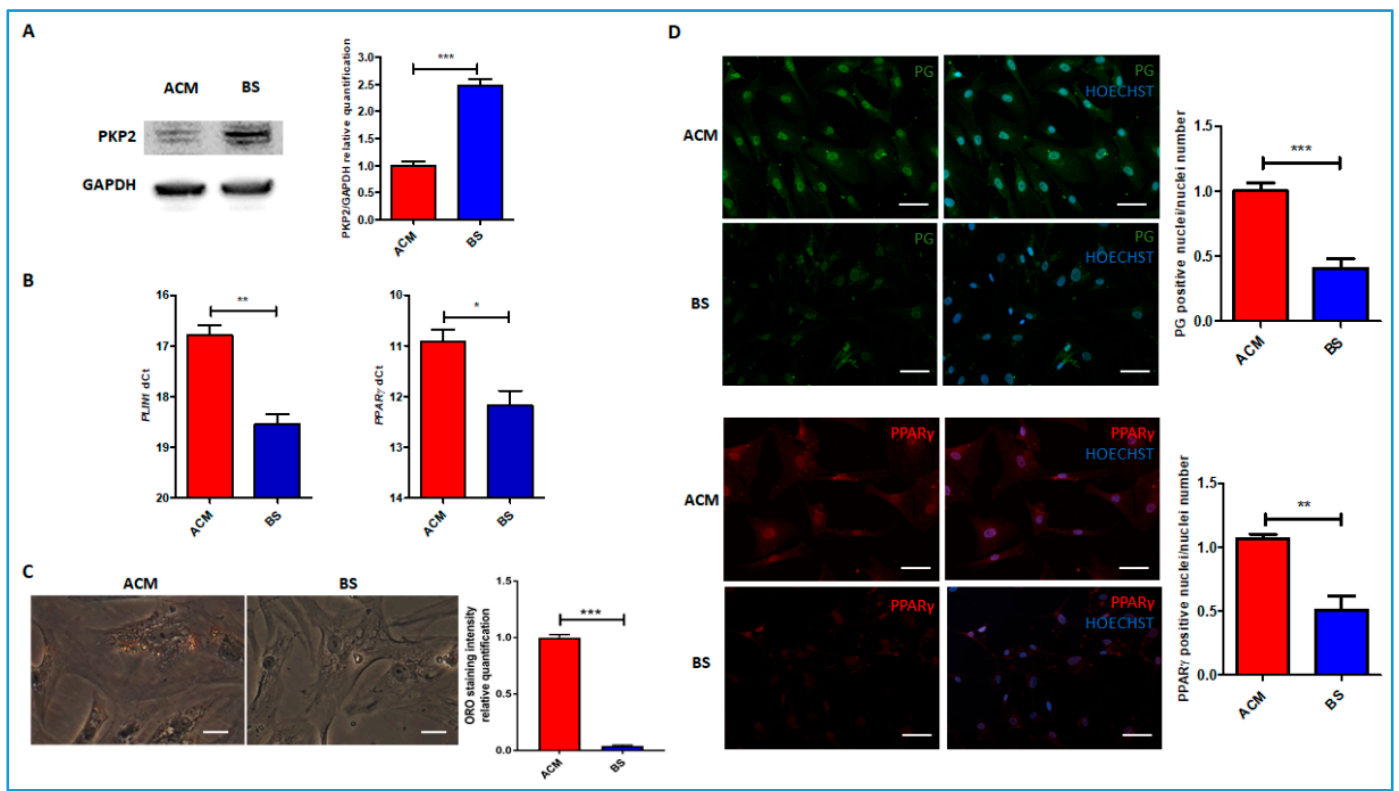

Figure 2. C-MSC data are distinctive of ACM. (A) Western blot shows higher PKP2 expression in ACM than in BS C-MSCs cultured in basal condition. GAPDH is shown as a loading control and used to normalize the quantification. (B) qRT-PCR data showing higher PLIN1 and PPAR $\gamma$ expression in ACM than in BS C-MSCs in adipogenic medium. (C) Higher ORO staining is detected in ACM vs. BS C-MSCs cultured in adipogenic medium for three days, as in the representative image and quantification. Scale bar is $20 \mu \mathrm{m}$. (D) Representative immunofluorescence images of C-MSCs cultured in adipogenic medium for three days and stained with PG or PPAR $\gamma$ antibodies. More positive nuclei/nuclei numbers were observed in ACM than in BS cells for both markers. Scale bar is $50 \mu \mathrm{m}$. The graphs show the mean and standard error of three technical replicates. ${ }^{*} p<0.05$; ${ }^{* *} p<0.01{ }^{* * *} p<0.001$ (two-tailed Student's $t$-test). ACM, arrhythmogenic cardiomyopathy; C-MSC, cardiac mesenchymal stromal cells; GAPDH, glyceraldehyde 3-phosphate dehydrogenase; ORO, Oil Red O; PG, plakoglobin; PKP2, plakophilin 2; PLIN1, perilipin-1; PPAR $\gamma$, peroxisome proliferator-activated receptor gamma.

We then investigated the capability of ACM and BS C-MSCs to accumulate lipids in adipogenic conditions. We found that ACM C-MSCs showed higher expressions of adipogenic genes such as PLIN1 and PPAR $\gamma$ and were more prone to accumulate neutral lipids than C-MSCs in the BS patient (Figure 2B, C). It has been reported that switching to adipogenic fate in ACM cases is linked to alterations in the Wnt/ $\beta$-catenin pathway and PG nuclear translocation [9]. ACM C-MSCs showed a larger number of PG-positive nuclei than BS C-MSCs as well as more PPAR $\gamma$ nuclear localization, as previously observed [14] when cultured in adipogenic medium (Figure 2D).

\section{Discussion}

Defining the meaning and the correct genotype-phenotype association of potentially pathogenic variants is an extreme challenge, even in the presence of in vitro functional validation. The effect of 
a mutation could be modified by various factors, such as the expression of the allele in different cell types, the patient's genetic background (which may include phenotype-modifier variants), and the compensation of other mechanisms, including epigenetic and environmental mechanisms.

To date, eight variants in the PKP2 gene-the most prevalent gene associated with ACM-have been found in BS patients, either alone or in combination with other gene variants [15-17]. In vitro studies in HL-1 cells of four of these variants showed sodium current alterations and a PKP2-dependent defect in sodium channel trafficking via microtubules to the intercalated disc [17]. We hypothesized that these mechanisms are shared between BS and ACM based on both also occurring in induced pluripotent stem cell (iPSC)-derived cardiomyocytes from a patient carrier of an ACM-associated PKP2 mutation and in PKP2-deficient mouse cardiomyocytes.

However, the present report describes one of the PKP2 BS-related mutations (p.S183N) associated with an ACM phenotype.

A limitation of the previous study [8] was the impossibility of determining whether the reported PKP2 mutation occurred in the context of additional genetic differences, other than in the four most common genes associated with BS. Indeed, there is a large number of known proteins that can directly or indirectly affect the sodium current. The clinical characteristics of the patient carrier of the p.S183N mutation were not definitely diagnostic of BS, since the type 1 ECG pattern was observed during a febrile episode and not confirmed by a provocative test [8]. This finding could therefore be an unspecific sign of BS-like ECG [18]. Further clinical investigation should be performed to exclude the presence of ACM in the concealed phase mimicking BS. The ClinGen consortium has reclassified most of the genes described as potentially linked to BS as not having sufficient evidence to support their causality for BS [19]. Thus, genetic testing of these genes may lead to incorrect interpretation of variants.

Despite being recognized as functionally relevant [8], rare (minor allele frequency (MAF) $=0.0082$ according to the Genome Aggregation Database) and relatively conserved among species, the available evidence cannot rule out a low pathogenicity effect of the PKP2 p.S183N variant alone. It may represent a phenotypic modulator with no clear deterministic cut-off through either BS or ACM phenotypes. Few rare causes of ACM have not been excluded by our genetic tests and known genetic causes of ACM only support about $50 \%$ of affected patients.

In conclusion, PKP2 variants in BS cases should be interpreted carefully. The complex decision-making network may benefit from additional functional analyses in cells that carry the whole genetic background of a patient, as in this present report, accompanied by a careful clinical and genetic interpretation in a family context.

Author Contributions: M.C. conceived and designed the research; G.P. and C.T. handled funding and supervision; S.P., C.A.P., A.S.M., I.S., A.R., and M.T. acquired the data; S.P., C.A.P., E.S., and M.C. drafted the manuscript; A.D.R., C.B., G.P., C.T., A.S.M., I.S., A.R, and M.T. critically revised the manuscript. E.S., A.D.R., and C.B. helped with data interpretation. All authors approved the final version of the manuscript and agree with all aspects of the work.

Funding: The project was funded by the Italian Ministry of Health (RC2019 EF5C ID:2754330).

Acknowledgments: We are grateful to patients who provided consent to participate to this study and to Matteo Pedrazzini for technical support.

Conflicts of Interest: C.T. receives consulting fees from Abbott, Medtronic, Boston Scientific, and Biosense Webster, and is member of EU Medtronic and Boston Scientific advisory boards. The other authors declare no relationships with industry.

\section{References}

1. Van Tintelen, J.P.; Entius, M.M.; A Bhuiyan, Z.; Jongbloed, R.; Wiesfeld, A.C.; Wilde, A.A.M.; Van Der Smagt, J.; Boven, L.G.; Mannens, M.; Van Langen, I.M.; et al. Plakophilin-2 Mutations Are the Major Determinant of Familial Arrhythmogenic Right Ventricular Dysplasia/Cardiomyopathy. Circulation 2006, 113, 1650-1658. [CrossRef] [PubMed]

2. Sieira, J.; Dendramis, G.; Brugada, P. Pathogenesis and management of Brugada syndrome. Nat. Rev. Cardiol. 2016, 13, 744-756. [CrossRef] [PubMed] 
3. Watanabe, H.; Minamino, T. Genetics of Brugada syndrome. J. Hum. Genet. 2015, 61, 57-60. [CrossRef] [PubMed]

4. Zhang, M.; Tavora, F.; Oliveira, J.; Li, L.; Franco, M.; Fowler, D.; Zhao, Z.; Burke, A. PKP2 mutations in sudden death from arrhythmogenic right ventricular cardiomyopathy (ARVC) and sudden unexpected death with negative autopsy (SUDNA). Circ. J. 2011, 76, 189-194. [CrossRef] [PubMed]

5. Catalano, O.; Antonaci, S.; Moro, G.; Mussida, M.; Frascaroli, M.; Baldi, M.; Cobelli, F.; Baiardi, P.; Nastoli, J.; Bloise, R.; et al. Magnetic resonance investigations in Brugada syndrome reveal unexpectedly high rate of structural abnormalities. Eur. Hear. J. 2009, 30, 2241-2248. [CrossRef] [PubMed]

6. Cerrone, M.; Delmar, M. Desmosomes and the sodium channel complex: Implications for arrhythmogenic cardiomyopathy and Brugada syndrome. Trends Cardiovasc. Med. 2014, 24, 184-190. [CrossRef]

7. Riele, A.S.T.; Agullo-Pascual, E.; A James, C.; Leo-Macias, A.; Cerrone, M.; Zhang, M.; Lin, X.; Lin, B.; Rothenberg, E.; Sobreira, N.L.; et al. Multilevel analyses of SCN5A mutations in arrhythmogenic right ventricular dysplasia/cardiomyopathy suggest non-canonical mechanisms for disease pathogenesis. Cardiovasc. Res. 2017, 113, 102-111. [CrossRef]

8. Cerrone, M.; Lin, X.; Zhang, M.; Agullo-Pascual, E.; Pfenniger, A.; Gusky, H.C.; Novelli, V.; Kim, C.; Tirasawasdichai, T.; Judge, D.P.; et al. Missense Mutations in Plakophilin-2 Cause Sodium Current Deficit and Associate with a Brugada Syndrome Phenotype. Circulation 2013, 129, 1092-1103. [CrossRef] [PubMed]

9. Sommariva, E.; Brambilla, S.; Carbucicchio, C.; Gambini, E.; Meraviglia, V.; Russo, A.D.; Farina, F.M.; Casella, M.; Catto, V.; Pontone, G.; et al. Cardiac mesenchymal stromal cells are a source of adipocytes in arrhythmogenic cardiomyopathy. Eur. Hear. J. 2015, 37, 1835-1846. [CrossRef] [PubMed]

10. Richards, S.; Aziz, N.; Bale, S.; Bick, D.; Das, S.; Gastier-Foster, J.; Grody, W.W.; Hegde, M.; Lyon, E.; On behalf of the ACMG Laboratory Quality Assurance Committee; et al. Standards and guidelines for the interpretation of sequence variants: A joint consensus recommendation of the American College of Medical Genetics and Genomics and the Association for Molecular Pathology. Genet. Med. 2015, 17, 405-423. [CrossRef] [PubMed]

11. Pilato, C.A.; Stadiotti, I.; Maione, A.S.; Saverio, V.; Catto, V.; Tundo, F.; Russo, A.D.; Tondo, C.; Pompilio, G.; Casella, M.; et al. Isolation and Characterization of Cardiac Mesenchymal Stromal Cells from Endomyocardial Bioptic Samples of Arrhythmogenic Cardiomyopathy Patients. J. Vis. Exp. 2018, e57263. [CrossRef] [PubMed]

12. Casella, M.; Pizzamiglio, F.; Russo, A.D.; Carbucicchio, C.; Al-Mohani, G.; Russo, E.; Notarstefano, P.; Pieroni, M.; D'Amati, G.; Sommariva, E.; et al. Feasibility of Combined Unipolar and Bipolar Voltage Maps to Improve Sensitivity of Endomyocardial Biopsy. Circ. Arrhythmia Electrophysiol. 2015, 8, 625-632. [CrossRef] [PubMed]

13. Marcus, F.I.; McKenna, W.J.; Sherrill, D.; Basso, C.; Bauce, B.; Bluemke, D.A.; Calkins, H.; Corrado, D.; Cox, M.G.; Daubert, J.P.; et al. Diagnosis of arrhythmogenic right ventricular cardiomyopathy/dysplasia: Proposed modification of the task force criteria. Circulation 2010, 121, 1533-1541. [CrossRef] [PubMed]

14. Dorn, T.; Kornherr, J.; Parrotta, E.I.; Zawada, D.; Ayetey, H.; Santamaria, G.; Iop, L.; Mastantuono, E.; Sinnecker, D.; Goedel, A.; et al. Interplay of cell-cell contacts and RhoA/ MRTF -A signaling regulates cardiomyocyte identity. EMBO J. 2018, 37, e98133. [CrossRef] [PubMed]

15. Forkmann, M.; Tomala, J.; Huo, Y.; Mayer, J.; Christoph, M.; Wunderlich, C.; Salmas, J.; Gaspar, T.; Piorkowski, C. Epicardial Ventricular Tachycardia Ablation in a Patient With Brugada ECG Pattern and Mutation of PKP2 and DSP Genes. Circ. Arrhythmia Electrophysiol. 2015, 8, 505-507. [CrossRef] [PubMed]

16. Allegue, C.; Coll, M.; Matés, J.; Campuzano, O.; Iglesias, A.; Sobrino, B.; Brion, M.; Amigo, J.; Carracedo, A.; Brugada, P.; et al. Genetic Analysis of Arrhythmogenic Diseases in the Era of NGS: The Complexity of Clinical Decision-Making in Brugada Syndrome. PLOS ONE 2015, 10, e0133037. [CrossRef] [PubMed]

17. Campuzano, O.; Fernández-Falgueras, A.; Iglesias, A.; Brugada, R. Brugada Syndrome and PKP2: Evidences and uncertainties. Int. J. Cardiol. 2016, 214, 403-405. [CrossRef] [PubMed]

18. Tsarouhas, K.; Papalexis, P.; Kafantaris, I.; Tsitsimpikou, C.; Vavetsi, S.; Rentoukas, E. Electrocardiographic findings compatible with Brugada syndrome in a patient with febrile respiratory infection. Hippokratia 2010, 14, 221-223. [PubMed]

19. Hosseini, S.M.; Kim, R.; Udupa, S.; Costain, G.; Jobling, R.; Liston, E.; Jamal, S.M.; Szybowska, M.; Morel, C.F.; Bowdin, S.; et al. Reappraisal of Reported Genes for Sudden Arrhythmic Death. Circulation 2018, 138, 1195-1205. [CrossRef] [PubMed]

(C) 2020 by the authors. Licensee MDPI, Basel, Switzerland. This article is an open access article distributed under the terms and conditions of the Creative Commons Attribution (CC BY) license (http://creativecommons.org/licenses/by/4.0/). 